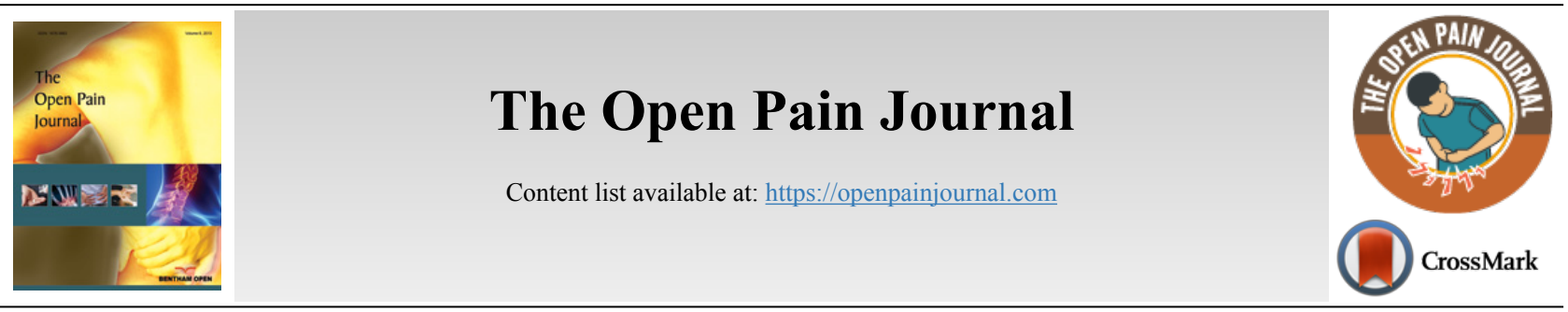

\title{
LETTER
}

\section{Minocycline Inhibits the Enhanced Antidromic Stimulation-induced Sensitization of C-Fibers Following Intradermal Capsaicin Injection}

\author{
Kerui Gong ${ }^{1,2}$ and Qing Lin ${ }^{1, *}$ \\ ${ }^{1}$ Department of Psychology, College of Science, University of Texas at Arlington, Arlington, Texas, USA \\ ${ }^{2}$ Department of Oral and Maxillofacial Surgery, University of California, San Francisco, California, USA
}

\begin{abstract}
:
Background:

Our previous studies indicated that retrograde signaling initiating from the spinal cord was mediated by the plasticity of Dorsal Root Ganglion (DRG) neurons. Both retrograde signaling and neuronal plasticity contributed to neurogenic inflammation, which were modulated by the activity of Satellite Glial Cells (SGCs). Thus, we want to know whether retrograde signaling is involved in the hypersensitivity of nociceptive afferents, and whether this process is affected by the plasticity of DRG neurons and glia.

Objective:

The study aims to examine if retrograde signaling can induce hypersensitivity of primary afferent nociceptors and if hypersensitivity involves glial modulation.

\section{Methods:}

Antidromic Electrical Stimulation (ES) of dorsal roots was used to mimic retrograde signaling activity. C- primary nociceptive afferent activity was recorded for testing the effect of antidromic ES. In a separate group, intradermal capsaicin injection to the ipsilateral hindpaw was used to prime DRG nociceptive neurons. For the third group, a glial inhibitor, minocycline, was pre-administered to test glial modulation in this process.

Results:

Antidromic ES sensitized the responses of C-fibers to nociceptive mechanical stimuli. For rats subjected to intradermal capsaicin injection, $\mathrm{C}$ fibers experienced more drastic sensitization induced by antidromic ES, shown as a greater response and longer duration, implying that sensitization correlates with the activation of DRG neurons.

Minocycline pretreatment significantly blocked the priming effect of capsaicin on C-fiber sensitization induced by antidromic ES, indicating the involvement of SGCs in DRG neuronal sensitization.

\section{Conclusion:}

Retrograde signaling may be one of the important mechanisms in neurogenic inflammation-mediated nociception, and this process is subjected to satellite glial modulation.
\end{abstract}

Keywords: Neurogenic inflammation, Antidromic electrical stimulation, Single fiber recording, C fiber, Capsaicin, Minocycline, Satellite glial cell.

\section{INTRODUCTION}

Inflammation-induced pain is one of the most common symptoms seen in a variety of inflammatory diseases, such as

\footnotetext{
* Address correspondence to this author at Department of Psychology, 501 South, University of Texas at Arlington, Arlington, TX 76019-0528, USA; Tel: +1-817-272-0154; Fax: +1-817-272-2364; E-mail: qilin@uta.edu
}

migraine, allergic arthritis, and inflammatory bowel diseases. Many studies demonstrate that the inflammatory process occurring in these diseases is mediated neurochemically, and is aptly termed neurogenic inflammation [1 - 4]. This process involves retrograde release of potent vasoactive neuropeptides, predominantly Calcitonin Gene-Related Peptide (CGRP) and substance $\mathrm{P}$ from activated peripheral nociceptive afferent 
terminals (usually $\mathrm{C}$ and $\mathrm{A} \delta$-fibers) leading to hyperemia and local edema [1 - 4]. It has been suggested that antidromic signaling along the primary afferent axons, by several mechanisms, triggers the retrograde release of these substances and the resulting hyperemia and local edema [5 - 9]. Among all the antidromic signaling processes, Dorsal Root Reflexes (DRRs) are suggested to be the most prominent mechanism [8, 10]. Using dorsal rhizotomy and neuropharmacology in an animal model of neurogenic inflammation by intradermal (i.d.) injection of Capsaicin (CAP), a series of our previous studies have strongly implied the existence of DRR-driven antidromic activity and its contributions to the incidence of neurogenic inflammation and sensitization of primary afferent nociceptors [11 - 14]. With the same model, we have further shown that antidromic Electrical Stimulation (ES) of dorsal roots, mimicking DRRs, consistently induced vasodilation in the hindpaw. This phenomenon was associated with the hyper excitability of nociceptive neurons in the Dorsal Root Ganglion (DRG), while the hyper excitability of neurons was subject to the modulation of Satellite Glial Cells (SGCs) [15, 16]. However, there is still a lack of direct evidence to demonstrate whether antidromic signaling in primary afferents induces hypersensitivity and/or sensitization of nociceptive afferent terminals.

Further, it would be very interesting to explore whether SGCs in the DRG are also involved in antidromic signalingevoked hypersensitivity of nociceptive afferent terminals that contribute to neurogenic inflammation-induced pain.

As a continuation of our preceding studies $[15,16]$, a serial of experiments in the present study were designed to examine whether retrograde signaling, mimicked by antidromic ES of dorsal roots, could induce hypersensitivity of primary afferent nociceptive activity, and whether the hypersensitivity is subjected to SGC's modulation. The completion of the study may help elucidate pain syndromes mediated by neurogenic inflammation, and point to new potential targets for controlling neurogenic inflammation.

\section{MATERIALS AND METHODS}

Male Sprague-Dawley rats weighing 280-320 g were used in this study. The animals were housed individually with food and water available ad libitum, and kept under controlled conditions of temperature $\left(23-25^{\circ} \mathrm{C}\right)$ and light/dark cycle $(12 \mathrm{~h} / 12 \mathrm{~h})$. All animals were handled in accordance with the protocol approved by the Institutional Animal Care and Use Committee at the University of Texas at Arlington.

\subsection{Drug Treatments}

Rats were randomly divided into three groups: saline + vehicle (Sal + Veh group), saline + capsaicin (Sal + CAP group) and minocycline + capsaicin (Min + CAP group). Minocycline hydrochloride (from Sigma-Aldrich) was diluted in saline and buffered to a $\mathrm{pH}$ of approximately 7.0, reaching a final concentration of $15 \mathrm{mg} / \mathrm{ml}$. Saline was used as control. CAP was dissolved into vehicle (7\% Tween 80 and $93 \%$ saline) with the final concentration of $1 \%$. Saline or minocycline (from Sigma-Aldrich) were administered intraperitoneally (i.p.) at $24 \mathrm{~h}$ before intradermal (i.d.) injection of vehicle or CAP. For Sal + Veh group, the rats received both i.p injection of saline and i.d injection of vehicle. For Sal + CAP group, the rats received both i.p injection of saline and i.d injection of CAP $(20 \mu \mathrm{l})$. For Min + CAP group, the rats received both i.p injection of minocycline $(30 \mathrm{mg} / \mathrm{kg})$ and i.d injection of CAP $(20 \mu \mathrm{l})$. Both doses of CAP and minocycline have been used in previous reports from our group [16, 17].

\subsection{Single C-fiber Recording}

Animals were first anesthetized with sodium pentobarbital $(50 \mathrm{mg} / \mathrm{kg})$ by i.p. injection, followed by a continuous i.v. infusion of the same anesthetic at a dose of $5-8 \mathrm{mg} / \mathrm{kg} / \mathrm{h}$ to maintain the anesthesia. The level of anesthesia was judged as being sufficient when the eye-blink reflex to air-puffs were absent. Once a stable level of anesthesia was reached, the animals were paralyzed with pancuronium (0.3-0.4 mg/h, i.v.) and artificially ventilated. Expiratory $\mathrm{CO}_{2}$ was monitored via a tracheal cannula and kept between $3.5-4.5 \%$. Core body temperature was monitored by a rectal probe and maintained near $37^{\circ} \mathrm{C}$ by a servo-controlled heating blanket. Single C-fiber recordings of afferent activity were performed using the established procedure described in detail in our previous studies $[14,17]$. In each rat, only one single fiber was recorded and used for analysis to avoid the desensitization or summation effect produced by multiple CAP injections. Briefly, the tibial nerve on the side of the hind limb that received CAP injection was exposed and kept in warm mineral oil. Only $1 / 4$ to $1 / 3$ of the tibial nerve was dissected out after a longitudinal cut, which would keep most parts of the tibial nerve undamaged so that the retrograde signals were still transmitted along the nerve to the periphery. The distal cut end of the tibial nerve was then teased into small filaments with fine-tipped forceps on a small mirror-based platform under a dissecting microscope until single-fiber activity of afferents from a fine nerve filament could be isolated on the basis of spike amplitude and waveform. The C-fiber was identified by conduction velocity $(<2 \mathrm{~m} / \mathrm{s})$ using the method established in our previous study [17].

Recorded action potentials and their responses to peripheral mechanical stimuli were amplified and displayed on a digital oscilloscope. Spikes were recorded and then converted and quantified by Spike-2 software (Cambridge Electronic Design).

Since mechanically evoked activity has been most commonly used to evaluate the sensitivity change in polymodal nociceptive axons (C-fibers) [18, 19], the present study conducted von Frey filament testing. Testing started with choosing an appropriate set of von Frey filaments by referencing to the "up and down method" [20]. According to the experience obtained from our previous work, a set of calibrated von Frey filaments with bending forces of 8.6, 14, 20 and $36 \mathrm{mN}$ were applied to the most sensitive spot of the receptive field of each C-fiber to evoke responses [14, 17]. Each filament was applied repetitively for $10 \mathrm{~s}$ at a frequency of about 2 strokes/s with enough force to cause slight bending and followed by a $10 \mathrm{~s}$ pause before the next filament was used to avoid a summation effect of the sensory stimulation leading to a false positive response. To minimize possible "human 
factor" bias, mechanical stimuli were applied without observation of the oscilloscope or computerized record so the experimenter was unaware of the response frequencies. Consistent with our previous reports [17, 21], all C-fibers identified and recorded responded to von Frey of 20 and 36 $\mathrm{mN}$, and firing rates increased in response to thicker von Frey filaments with greater bending force. Thus, these fibers were considered to be nociceptive units according to a previous report [18]. In our initial study, we first examined whether antidromic ES of dorsal roots influenced firing frequencies of C-fibers evoked by graded mechanical stimuli.

For antidromic ES of dorsal roots, the L4 and L5 dorsal roots were exposed by laminectomies and their identities were confirmed by their relations to the corresponding vertebra. Dorsal rhizotomies were then performed on the ipsilateral side where single fiber activity was recorded [11, 13]. A bipolar silver electrode was placed on the cut distal ends of dorsal rootlets. The parameters for antidromic ES were $10 \mathrm{~Hz}, 1 \mathrm{~ms}$, and $3 \mathrm{~mA}$, which have been shown to activate C-fibers [15, 22].

CAP was i.d. injected into the glabrous skin of one hind paw to evoke neurogenic inflammation as reported previously by our group [11, 13-16]. Two hours later, a C-fiber with a receptive field adjacent to, but out of the CAP injection site (about $3 \mathrm{~mm}$ away from the edge of the receptive field), was picked and recorded, which would minimize the local effect of CAP injection, out of the range of CAP diffusion from the injection site. For the control group, similar procedures were performed except the animals received i.d. injection of vehicle used to dissolve CAP (7\% Tween 80 and $93 \%$ saline). To minimize possible experimental bias, the experimenters were not aware whether CAP or vehicle was injected to the animal being recorded. After the fiber was chosen, baseline activity was recorded followed by $10 \mathrm{~min}$ of antidromic ES. Then the sensitized effect of antidromic ES was observed at 0, 5, 15 and 30 min after antidromic ES.

\subsection{Statistical Analysis}

Since antidromic activation of dorsal roots unavoidably involves excitation of DRG Neurons, we further tested whether plastic changes of DRG neurons contributed to the antidromic ES-produced sensitization of primary nociceptive afferents. To achieve this, we used intradermal CAP injection to prime DRG neurons in the Sal + CAP group. We have used two ways to avoid the local effect of CAP on peripheral endings of primary afferents: 1) fibers with receptive fields away from CAP injection site were picked and recorded as described in "MATERIALS AND METHODS"; and 2) tests were conducted $2 \mathrm{~h}$ after CAP injection. The latter was based on our previous study that demonstrates that primary sensitization of nociceptive afferents lasts up to $60 \mathrm{~min}$ after CAP injection percentage changes from the pre-antidromic ES value. Statistical significance was tested using one-way ANOVA with repeated measures followed by a Bonferroni-corrected t-test in the same group to compare differences between the baseline (pre-CAP injection) and responses after injection at different time points. One-way ANOVA followed by a StudentNewman-Keuls test was used to compare the differences in responses between groups having different treatments at the same time point. A p-value of less than 0.05 was regarded as significant. All values were expressed as mean \pm SEM.

\section{RESULTS}

\subsection{Antidromic ES of Dorsal Roots Induced Sensitization of Primary Nociceptive Afferents}

In vehicle treated animals (Sal + Veh group), 12 fibers of $15(80 \%)$ showed enhanced responses to von Frey filaments with bending forces of 20 and $36 \mathrm{mN}$ following antidromic ES. Forces of 8.6 and $14 \mathrm{mN}$ did not evoke any significant responses. As demonstrated in Fig. (1A), before antidromic ES the firing frequency in response to von Frey stimuli was 2.0 and $3.1 \mathrm{~Hz}$ respectively. The firing was increased to 3.3 and $7.2 \mathrm{~Hz}$ immediately after antidromic ES. Pooled data showed that firing frequency evoked by $20 \mathrm{mN}$ at this time was significantly higher than baseline $(100 \%$ v.s. $137.2 \pm 14.3 \%, \mathrm{p}$ $<0.05$, Fig. (1B)). For the firing frequency evoked by $36 \mathrm{mN}$ following antidromic ES, a greater increase was discerned, which increased to $169.2 \pm 29.3 \%$ of baseline $(p<0.05, n=12$, Fig. (1B)). A time related observation was also made on the effects of antidromic ES on mechanical-evoked firing of fibers. As shown in Fig. (1B), at 0 and 5 min after antidromic ES, the firing frequency induced by $20 \mathrm{mN}$ was increased to $137.2 \pm$ $14.3 \%$ and $131.5 \pm 23.5 \%$, respectively, and both were significantly higher than baseline.

However, the firing frequency kept decreasing over the time. At 15 and $30 \mathrm{~min}$ after ES, the firing frequency decreased to $110.5 \pm 12.7 \%$ and $105.6 \pm 16.6 \%$ respectively. In contrast, for the effect of ES on responses to $36 \mathrm{mN}$, the scenario was similar but the responses were much greater and lasted longer, up to $15 \mathrm{~min}$. The firing rate induced by von Frey stimuli increased to $169.2 \pm 31.3 \%$ of baseline at 0 min after antidromic ES, $163.3 \pm 28.8 \%$ at $5 \mathrm{~min}(\mathrm{p}<0.01, \mathrm{n}=12)$, and $159.5 \pm 19.2 \%$ at $15 \min (\mathrm{p}<0.05, \mathrm{n}=12$; compared with baseline, Fig. (1B)). At $30 \mathrm{~min}$, the responses did not show significant difference with baseline $(108.3 \pm 16.6 \%, \mathrm{p}>0.05, \mathrm{n}$ $=12$ ). Therefore, in the following experiments, no observation was conducted at time points later than 15 min after ES. Overall, these results demonstrated that antidromic ES enhances nociceptive mechanical responses with a preference for stronger mechanical stimuli. 


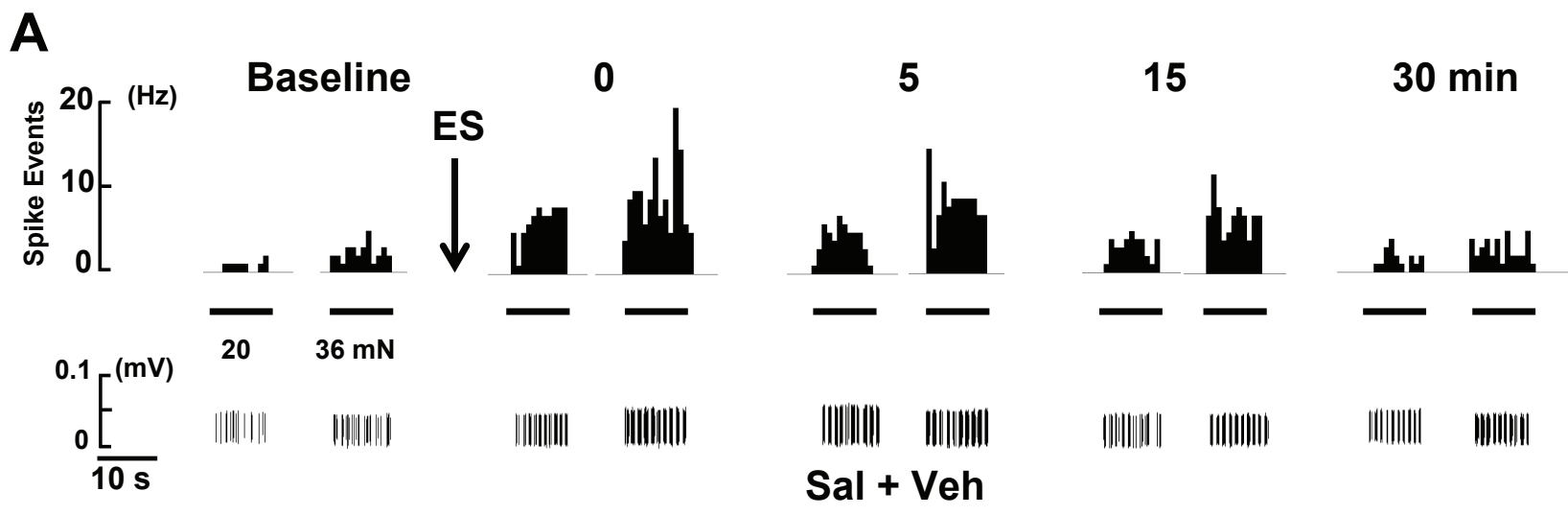

B

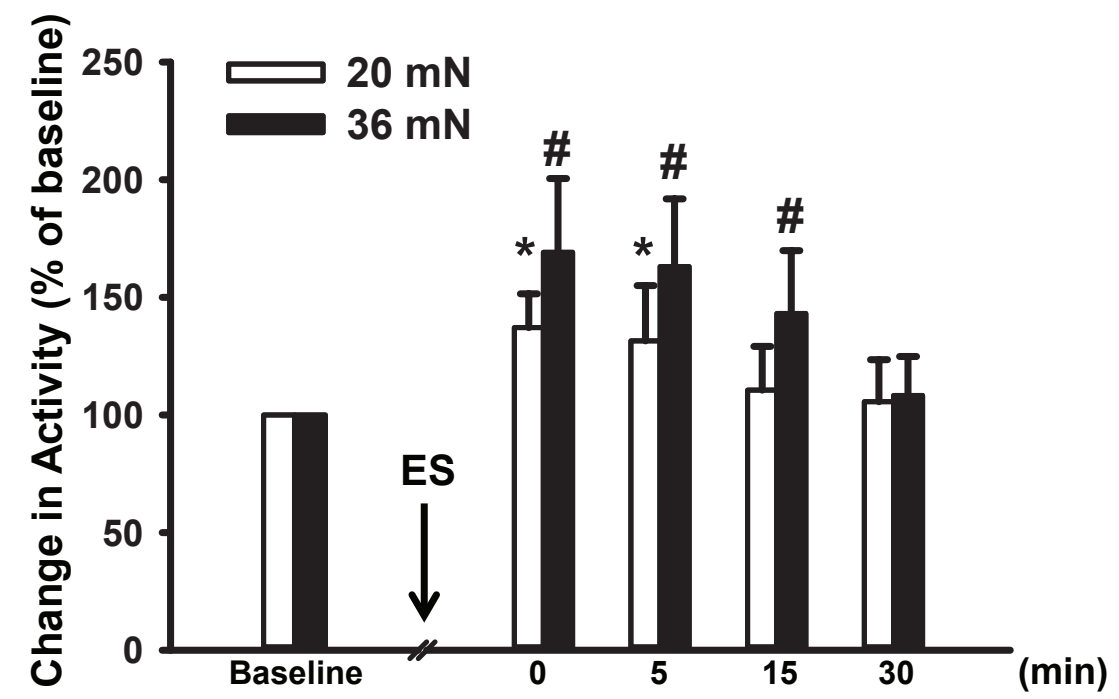

Fig. (1). Antidromic Electrical Stimulation (ES) of dorsal roots induced sensitization of peripheral C fibers. A. Representative traces showed the firing of a single fiber induced by von Frey filaments at baseline, 0, 5, 15 and $30 \mathrm{~min}$ after antidromic ES of dorsal roots. Upper panel: the real-time histogram showing the spike frequency of the fiber with von Frey stimulation. Lower panel: original firing traces from the fiber. B. Pooled data from 12 fibers of 12 rats showed antidromic ES-induced sensitization in firing of fibers to von Frey stimuli. Sensitization exhibited longer duration when the fibers were challenged with stronger intensity. *, p $<0.05$; responses after ES $v$ s. before ES with the stimulus intensity of $20 \mathrm{mN}$. \#, p $<0.05$ responses after ES $v s$ before ES with the stimulus intensity of $36 \mathrm{mN}$.

\subsection{Antidromic ES-induced Sensitization of Primary Nociceptive Afferents Was Enhanced Following CAP Injection}

Since antidromic activation of dorsal roots unavoidably involves excitation of DRG Neurons, we further tested whether plastic changes of DRG neurons contributed to the antidromic ES-produced sensitization of primary noci-ceptive afferents. To achieve this, we used intradermal CAP injection to prime DRG neurons in the Sal + CAP group. We have used two ways to avoid the local effect of CAP on peripheral endings of primary afferents: 1) fibers with receptive fields away from CAP injection site were picked and recorded as described in "MATERIALS AND METHODS"; and 2) tests were conducted $2 \mathrm{~h}$ after CAP injection. The latter was based on our previous study that demonstrates that primary sensi-tization of nociceptive afferents lasts up to $60 \mathrm{~min}$ after CAP injection
[17]. In addition, minimal local effect of CAP was confirmed with the observation that the firing frequency of C-fibers evoked by von Frey filaments of 20 and $36 \mathrm{mN}$ at $2 \mathrm{~h}$ after CAP injection was similar to that in control groups. The firing for $\mathrm{C}$-fibers to stimuli of 20 and $36 \mathrm{mN}$ at baseline was $1.9 \pm$ $0.5 \mathrm{~Hz}$ and $3.4 \pm 0.4 \mathrm{~Hz}$, respectively, in the Sal + CAP group, while the responses were $2.1 \pm 0.2 \mathrm{~Hz}$ and $3.1 \pm 0.3 \mathrm{~Hz}$ in the $\mathrm{Sal}+$ Veh group $(p>0.05, \mathrm{n}=16$ for Sal + CAP group, and $\mathrm{n}$ $=12$ for Sal + Veh group). This confirmed that at this time point, primary sensitization induced by CAP has decreased to the minimal effect.

In the Sal + CAP group (Fig. 2), 16 out of 18 fibers showed enhanced mechanical-evoked responses following antidromic ES, while the remaining two showed no changes. Statistical analysis did not reveal a significant difference in the responsive percentage of the two groups ( $p>0.05$, Chi square, data not shown). 
A

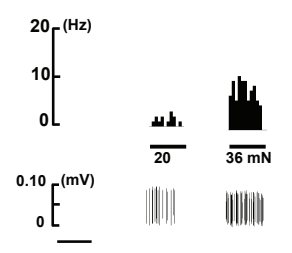

B

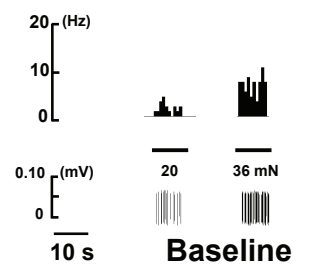

Sal + CAP<smiles>S=S</smiles><smiles>S=S</smiles>

Min + CAP
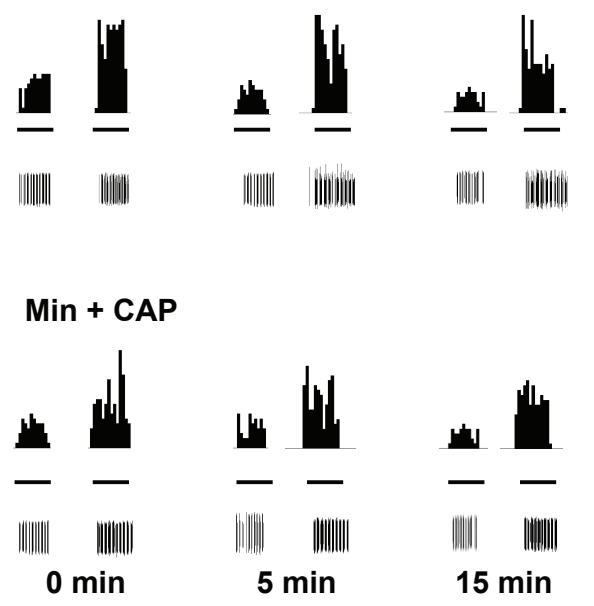

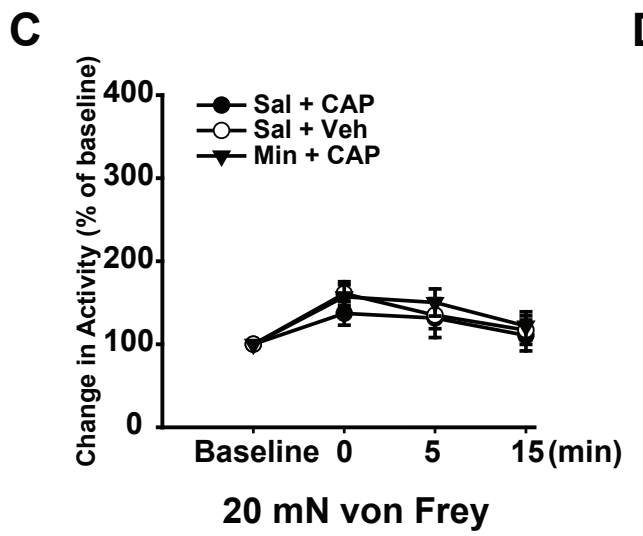

D

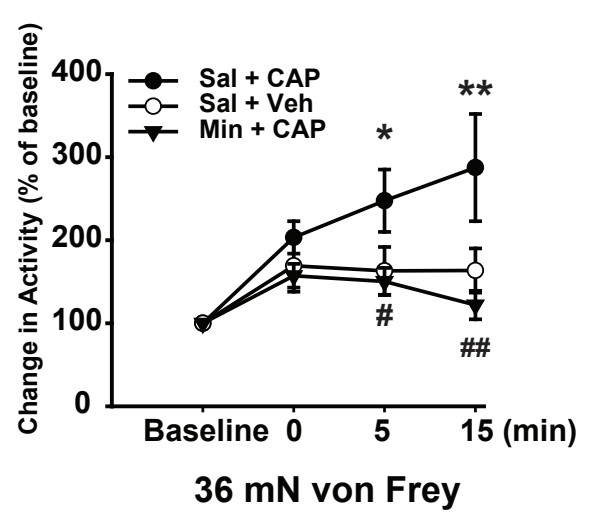

Fig. (2). Intradermal injection of CAP $(2 \mathrm{~h})$ enhanced the sensitization of $\mathrm{C}$ fibers induced by antidromic ES, while minocycline pretreatment completely blocked the enhancement induced by CAP. A. Representative traces showed the firing of a single fiber at baseline, 0,5 , and 15 min after antidromic ES of dorsal roots. Upper panel: the real- time histogram showing the spike frequency of the fiber with von Frey stimulation. Lower panel: original firing traces from the fiber. B. Representative traces showed the firing of a single fiber at baseline, 0, 5, and 15 min after antidromic ES of dorsal roots. The animal received minocycline $24 \mathrm{~h}$ before CAP injection. Upper panel: the real- time histogram showing the spike frequency of the fiber with von Frey stimulation. Lower panel: original firing traces from the fiber. C. Statistical analysis showed that for firing of C fibers induced by $20 \mathrm{mN}$ von Frey, no significant difference in antidromic ES-induced enhancement was detected among the 3 groups. D. Statistical analysis showed that firing of $\mathrm{C}$ fibers induced by $36 \mathrm{mN}$ von Frey was dramatically increased; while at all the three time points, minocycline showed complete inhibition. *, p $<0.05 ; * *, p<0.01 ;$ Sal + Veh group compared with Veh + CAP group. \#,p $<0.05$ and \#\#,p $<0.01$, Min + CAP group compared with Veh + CAP group.

For the firing frequency induced by $20 \mathrm{mN}$ in the Sal + CAP group (Fig. 2A and C), immediately following antidromic ES $(0 \mathrm{~min})$ the firing frequency increased to 161.0 $\pm 14.3 \%$ of baseline ( $p<0.05$, compared to baseline of the same group). At $5 \mathrm{~min}$ after antidromic ES, the firing frequency was $135.0 \pm 16.3 \%$, and both were significantly higher than baseline. The enhancement of firing after antidromic ES was then compared between Sal + Veh and Sal + CAP groups. For the magnitude of enhancement, the Sal + CAP group showed a tendency to be higher for the first two time points ( 0 and $5 \mathrm{~min}$ ) compared with the $\mathrm{Sal}+\mathrm{Veh}$ group. However, the differences between groups were not significant in these or any of the remaining times points (Fig. 2C).

For the responses induced by $36 \mathrm{mN}$, the $\mathrm{Sal}+\mathrm{CAP}$ group showed much greater changes. Immediately after antidromic ES, the firing frequency was increased to $203.4 \pm 18.9 \%(p<$ 0.01 , compared with baseline of the same group, Fig. (2A and
D). At 5 min after antidromic ES, the firing frequency induced by $36 \mathrm{mN}$ was even higher with the value of $247.6 \pm 37.6 \%$. We witnessed the largest sensitizing effect at $15 \mathrm{~min}$ post antidromic ES, which reached $287.5 \pm 64.6 \%$ of the baseline. The magnitude of enhancement after antidromic ES was then compared between $\mathrm{Sal}+\mathrm{Veh}$ and $\mathrm{Sal}+\mathrm{CAP}$ groups. No significance was discerned between the two groups at $0 \mathrm{~min}$ after antidromic ES. However, at 5 min after antidromic ES, the fibers in the Sal + CAP group showed a stronger sensitization than the Sal + Veh group. The magnitude of enhancement in the Sal + CAP group was $247.6 \pm 27.6 \%$ of baseline, while it was only $163.3 \pm 28.8 \%$ in the Sal + Veh group $(p<0.05$, Fig. 2D). A similar change was observed at the 15 minute time point, i.e. the antidromic ES-induced sensitization was $287.5 \pm 34.6 \%$ of baseline in the Sal + CAP group, while the value was $159.5 \pm 19.2 \%$ in $\mathrm{Sal}+\mathrm{Veh}$ group $(p<0.01$, Fig. 2D). These revealed that the time course of 
sensitization induced by antidromic ES lasted much longer in $\mathrm{Sal}+\mathrm{CAP}$ group than Sal + Veh group (15 min $v s 5 \mathrm{~min})$, strongly indicating that CAP injection has an additive effect on antidromic ES-induced sensitization of $\mathrm{C}$ nociceptive afferent fibers, possibly by modulating DRG neuronal plasticity.

\subsection{Enhancement of Antidromic ES-induced Sensitization Following CAP Injection was Inhibited by Minocycline Pretreatment}

Our previous study shows that inhibition of SGCs by minocycline reduced the neurogenic inflammation induced by antidromic ES of dorsal roots following CAP injection, strongly indicating the involvement of SGCs in modulating sensitization of DRG neuronal activity following CAP injection $[15,16]$. Therefore, we wanted to further investigate whether such a modulatory effect of SGCs is also involved in the enhancement of antidromic ES-induced sensitization caused by CAP injection.

Minocycline was injected $24 \mathrm{~h}$ before CAP injection. Firstly we compared the firing of C-fibers at baseline to see whether there was any effect of minocycline at this time point. At baseline, the firing of C-fibers was $2.0 \pm 0.7 \mathrm{~Hz}$ (before antidromic ES with $20 \mathrm{mN}$ ) and $3.1 \pm 0.6$ (before antidromic ES with $36 \mathrm{mN}$ ) respectively, which showed no significant difference compared with animals receiving CAP injection only $(\mathrm{Sal}+\mathrm{CAP})$. Following antidromic ES, 15 out of 19 fibers in minocycline group (Min + CAP) showed enhanced mechanical-evoked responses.

Statistical analysis did not reveal a significant difference in the responsive percentage compared with the other two groups ( $p>0.05$, Chi square, data not shown). In the Min + CAP group, the firing frequency increased to $3.0 \pm 0.4$ (induced by $20 \mathrm{mN}$ ) and $4.7 \pm 0.8$ (induced by $30 \mathrm{mN}$ ) immediately following antidromic ES. This sensitization effect was prominent until $15 \mathrm{~min}$ following antidromic ES.

We further compared the increased percentage in firing following antidromic ES in all three groups. However, as for the sensitization effect induced by antidromic ES, $20 \mathrm{mN}$ von Frey stimuli did not produce a significant difference in amplitude among three groups (Fig. 2C); while the response of C-fibers to $36 \mathrm{mN}$ von Frey showed a different pattern. Minocycline significantly inhibited the CAP-induced enhancement of $36 \mathrm{mN}$-evoked responses (Fig. 2D). At $0 \mathrm{~min}$ following antidromic $\mathrm{ES}$, the enhancement of $\mathrm{C}$ fiber firing to $36 \mathrm{mN}$ was $157.2 \pm 14.3 \%$ of the baseline, which showed a tendency to be lower than CAP only group $(\mathrm{Sal}+\mathrm{CAP})$, but no significant difference were detected among three groups (Fig. 2D). At $5 \mathrm{~min}$, the enhancement dropped to $150.3 \pm 17.1 \%$, which was significantly lower than the CAP only group $(\mathrm{p}<$ 0.05 ). This suggested that minocycline showed an inhibitory effect on the enhancement of antidromic ES-induced sensitization. At $15 \mathrm{~min}$, the inhibitory effect was even more obvious. The enhancement was $121.9 \pm 12.8 \%$, which was significantly lower than CAP only group $(287.5 \pm 64.3 \%$, p < $0.01)$ and comparable to the saline group $(159.5 \pm 26.7 \%)$. Overall, these results provide evidence that DRG neurons are sensitized following CAP injection and this process is subject to SGC's modulation.

\section{DISCUSSION}

The main findings of the current study are that sensitization of C-fibers can be induced by antidromic ES of dorsal roots which mimicked DRRs in neurogenic inflammation. Furthermore, sensitization was enhanced following CAP injection by producing a priming effect on DRG neurons. Interestingly, the enhanced sensitization was largely blocked by a glial inhibitor minocycline. Thus, the study provided further evidence that sensitization of primary nociceptors is associated with spinally-mediated retrograde signaling and the plasticity of DRG neurons, while all these processes are subject to SGC's modulation in the DRG.

Retrograde signaling along primary nociceptive $\mathrm{C}$ (or A $\delta$ ) fibers has been established to contribute to neurogenic inflammation by our and other groups [5, 9, 11 - 13, 15]. However, it still remains inconsistent as to whether antidromic ES of primary afferents induces sensitization of primary afferent nociceptors [23-27]. By using antidromic ES of dorsal roots, the results of the present study clearly point out that antidromic activation of primary afferent neurons and their axons induced sensitization of C-nociceptive fibers to mechanical stimulation. In fact, we think there are several differences contributing to the discrepancy. Firstly, in some reports, the authors focused on testing the thermal sensitivity of C-fibers, leaving mechanical sensitivity unchecked [26, 27]. So, it is unknown whether mechanical hyperalgesia was affected in their models. Secondly, there is a distinct difference among the preparations. In the other studies, the peripheral nerves (either saphenous or sural nerves) were stimulated antidromically, while in our present study dorsal roots were stimulated antidromically. The biggest difference between these two preparations is that DRG neuronal cell bodies were activated when dorsal roots were stimulated, while DRG neuronal cell bodies were largely untouched in the peripheral nerve stimulation scenario used in other reports. DRG neuronal cell bodies are the main pool for receptors and mediators to be stored and released.

Therefore, it is likely that antidromic ES of dorsal roots sensitizes the DRG neuronal cell bodies leading to the release of some inflammatory mediators like CGRP and prostaglandin E2 from the primary afferent terminals, contributing to the mechanical hypersensitivity [7]. This view is strongly supported by our previous studies [13 - 15]. Thirdly, in our current study we used stimulating parameters different from those used by these other groups. The parameters for antidromic ES of dorsal roots $(10 \mathrm{~Hz}, 1 \mathrm{~ms}$ and $3 \mathrm{~mA}$ for 10 $\mathrm{min}$ ) in the current study have been widely used and proven to be an effective and reliable means for activating C-fibers [15, $22,28]$. Therefore, the data obtained from the current study more reliably indicate that retrograde signaling contributed to mechanical hyperalgesia in neurogenic inflammation. However, we did notice that for each group about $10 \%$ of Cfibers showed either no response or decreased responses to antidromic ES (data not shown). The detailed mechanism is unclear but we think this was likely due to a deteriorative change in the preparation, especially since we noticed this phenomenon more at the beginning of the project when the experimenters were gaining technical proficiency. 
Another important finding in the present study is that priming the animals with CAP greatly enhanced the antidromic ES-induced sensitization of C-fibers. In the Sal + CAP group, the antidromic ES-induced sensitization was much larger and the duration was much longer ( $>30 \mathrm{~min}$ ) compared with control group (15 min). This enhancement was unlikely due to the peripheral sensitization produced by CAP injection because our previous report has already shown that peripheral sensitization induced by CAP injection lasted no more than $1 \mathrm{~h}$ [17]. To address this concern, we tested the effect of antidromic ES at $2 \mathrm{~h}$ after CAP injection. We also carefully chose the fibers recorded with their receptive fields out of sensitized area. Our data showed that the baseline for C fiber firing before antidromic ES did not differ between groups of vehicle and CAP treatments. Therefore, enhanced antidromic ES- induced sensitization following CAP injection should not be mainly due to the sensitization of fibers or its terminals, but is most likely related to the neuronal excitability residing in the DRG. Recently, some reports showed that DRG neurons could be primed and this might be important for the switch between acute and long-lasting pain [29].

Another line of evidence supporting this is that our results showed that antidromic ES of dorsal roots induced sensitization while stimulation of peripheral nerves did not induce such a change [26, 27], suggesting the important role of DRG neuronal soma in the process. Antidromic ES-induced C-fiber sensitization has a preference for nociceptive stimuli (20 and $36 \mathrm{mN})$ but not the non-nociceptive one $(8.4 \mathrm{mN})$. Notably, compared with the $\mathrm{Sal}+\mathrm{Veh}$ group, the Sal + CAP group showed a larger enhancement for firing induced by $36 \mathrm{mN}$ than that by $20 \mathrm{mN}$. These changes indicate that antidromic ES had more effect on the nociceptive stimuli, which further demonstrated the important role the hyperactive DRG neurons play in peripheral sensitization by the mechanism of neurogenic inflammation.

Our third finding is that minocycline almost completely blocked the enhancement of antidromic ES-induced sensitization following CAP injection. This is supported by our previous work in which minocycline reduced the CAP-induced enhancement of neuronal excitability of DRG neurons and the resulting neurogenic inflammatory vasodilation $[15,16]$. Minocycline has been widely used as a glial inhibitor and proved to be glial specific with the current dose of $30 \mathrm{mg} / \mathrm{kg}$ [30]. Therefore, the results from the current study provide direct evidence of glial cell involvement in CAP-induced neurogenic inflammation by affecting both vasodilation [15] and afferent sensitization (Fig. 2). Since dorsal roots were cut and the DRG was included in our preparation, the glial cells potentially involved should be SGCs, as they are the predominant glial cells in DRGs [31]. Based on this, it is plausible that minocycline affects the process of sensitization through its actions on SGCs as suggested by our previous work [16]. Considering their close proximity with neurons, SGCs are definitely involved in modulating DRG neurons [31].

SGCs can release a variety of cytokines or substances once activated, such as tumor necrosis factor- $\alpha(\mathrm{TNF} \alpha)$, glutamate, adenosine triphosphate, etc [32-34]. These molecules can affect the neuronal excitability of the DRG $[15,16]$. While our current work did not identify which molecules are involved, our previous work strongly indicated that TNF $\alpha$ is one of the key factors involved in the plasticity of DRG neurons in the process of neurogenic inflammation [16]. In the scenario of the present study, we propose that minocycline inhibits the activation of SGCs, reducing the release of certain cytokines such as TNF $\alpha$. Thus, TNF $\alpha$ is likely serving as a "medium" for the mutual activation between SGCs and DRG neurons [35].

\section{CONCLUSION}

The present study provides direct evidence showing that antidromic activity-mediated retrograde signaling in the spinal cord facilitates sensitization of primary nociceptive afferents in the course of neurogenic inflammation. The study also indicates that the plasticity of nociceptive neurons in the DRG contributes to this process. One of the underlying mechanisms is satellite glial modulation.

\section{LIST OF ABREVIATIONS}

$\begin{array}{lll}\text { CAP } & = & \text { Capsaicin } \\ \text { DRG } & = & \text { Dorsal Root Ganglion } \\ \text { DRRs } & = & \text { Dorsal Root Reflexes } \\ \text { ES } & = & \text { Electrical Stimulation } \\ \text { I.D. } & = & \text { Intradermal } \\ \text { I.P. } & = & \text { Intraperitoneal } \\ \text { SGCs } & = & \text { Satellite Glial Cells } \\ \text { TNFa } & = & \text { Tumor Necrosis Factor- } \alpha\end{array}$

\section{ETHICS APPROVAL AND CONSENT TO PARTICIPATE}

All animals were handled in accordance with the protocol approved by the Institutional Animal Care and Use Committee at the University of Texas at Arlington.

\section{HUMAN AND ANIMAL RIGHTS}

The reported experiments are in accordance with the standards of The US Public Health Service's "Policy on Humane Care and Use of Laboratory Animals," and "Guide for the Care and Use of Laboratory Animals.

\section{CONSENT FOR PUBLICATION}

All participants provided written informed consent to participate.

\section{FUNDING}

This work is supported by NIH grant NS040723 awarded to Qing Lin.

\section{CONFLICT OF INTEREST}

The authors declare no conflict of interest, financial or otherwise.

\section{ACKNOWLEDGEMENTS}

The authors would like to thank Michael Quast, Ph.D. for his helpful editing of the English language while preparing the manuscript. 


\section{REFERENCES}

[1] Herbert MK, Holzer P. Neurogenic inflammation. II. pathophysiology and clinical implications. Anasthesiol Intensivmed Notfallmed Schmerzther 2002; 37(7): 386-94.

[http://dx.doi.org/10.1055/s-2002-32701] [PMID: 12101511]

[2] Richardson JD, Vasko MR. Cellular mechanisms of neurogenic inflammation. J Pharmacol Exp Ther 2002; 302(3): 839-45. [http://dx.doi.org/10.1124/jpet.102.032797] [PMID: 12183638]

[3] Geppetti P, Rossi E, Chiarugi A, Benemei S. Antidromic vasodilatation and the migraine mechanism. J Headache Pain 2012; 13(2): 103-11

[http://dx.doi.org/10.1007/s10194-011-0408-3] [PMID: 22200764]

[4] Malhotra R. Understanding migraine: Potential role of neurogenic inflammation. Ann Indian Acad Neurol 2016; 19(2): 175-82. [http://dx.doi.org/10.4103/0972-2327.182302] [PMID: 27293326]

[5] Jänig W, Lisney SJ. Small diameter myelinated afferents produce vasodilatation but not plasma extravasation in rat skin. J Physiol 1989; 415: 477-86.

[http://dx.doi.org/10.1113/jphysiol.1989.sp017732] [PMID: 2640468]

[6] Häbler HJ, Timmermann L, Stegmann JU, Jänig W. Involvement of neurokinins in antidromic vasodilatation in hairy and hairless skin of the rat hindlimb. Neuroscience 1999; 89(4): 1259-68.

[http://dx.doi.org/10.1016/S0306-4522(98)00322-4] [PMID: 10362 313]

[7] Kress M, Guthmann C, Averbeck B, et al. Calcitonin gene-related peptide and prostaglandin E2 but not substance $\mathrm{P}$ release induced by antidromic nerve stimulation from rat skin in vitro. Neuroscience 1999; 89: 303-10.

[8] Willis WD. Dorsal root potentials and dorsal root reflexes: A doubleedged sword. Exp Brain Res 1999; 124: 395-421.

[9] Carmichael NM, Dostrovsky JO, Charlton MP. Enhanced vascular permeability in rat skin induced by sensory nerve stimulation: evaluation of the time course and appropriate stimulation parameters. Neuroscience 2008; 153(3): 832-41.

[http://dx.doi.org/10.1016/j.neuroscience.2008.02.068] [PMID: 1842 0352]

[10] Cervero F, Laird JM, García-Nicas E. Secondary hyperalgesia and presynaptic inhibition: An update. Eur J Pain 2003; 7(4): 345-51. [http://dx.doi.org/10.1016/S1090-3801(03)00047-8] [PMID: 12821 405]

[11] Lin Q, Wu J, Willis WD. Dorsal root reflexes and cutaneous neurogenic inflammation after intradermal injection of capsaicin in rats. J Neurophysiol 1999; 82(5): 2602-11

[http://dx.doi.org/10.1152/jn.1999.82.5.2602] [PMID: 10561430]

[12] Lin Q, Zou X, Willis WD. Adelta and C primary afferents convey dorsal root reflexes after intradermal injection of capsaicin in rats. $\mathrm{J}$ Neurophysiol 2000; 84(5): 2695-8. [http://dx.doi.org/10.1152/jn.2000.84.5.2695] [PMID: 11068011]

[13] Lin Q, Li D, Xu X, Zou X, Fang L. Roles of TRPV1 and neuropeptidergic receptors in dorsal root reflex-mediated neurogenic inflammation induced by intradermal injection of capsaicin. Mol Pain 2007; $3: 30$

[http://dx.doi.org/10.1186/1744-8069-3-30] [PMID: 17961222]

[14] Li D, Ren Y, Xu X, Zou X, Fang L, Lin Q. Sensitization of primary afferent nociceptors induced by intradermal capsaicin involves the peripheral release of calcitonin gene-related Peptide driven by dorsal root reflexes. J Pain 2008; 9(12): 1155-68.

[http://dx.doi.org/10.1016/j.jpain.2008.06.011] [PMID: 18701354]

[15] Gong K, Zou X, Fuchs PN, Lin Q. Minocycline inhibits neurogenic inflammation by blocking the effects of tumor necrosis factor- $\alpha$. Clin Exp Pharmacol Physiol 2010; 482: 177-81.

[16] Gong K, Zou X, Fuchs NP. Minocycline inhibits neurogenic inflammation by blocking the effects of tumor necrosis factor- $\alpha$. Clin Exp Pharmacol Physiol 2015; 42: 940-9.

[http://dx.doi.org/10.1111/1440-1681.12444] [PMID: 26175075]

[17] Ren Y, Zou X, Fang L, Lin Q. Sympathetic modulation of activity in Adelta- and C-primary nociceptive afferents after intradermal injection of capsaicin in rats. J Neurophysiol 2005; 93(1): 365-77. [http://dx.doi.org/10.1152/jn.00804.2004] [PMID: 15371497]

[18] Leem JW, Willis WD, Chung JM. Cutaneous sensory receptors in rat foot. J Neurophysiol 1993; 69:1684-99.

[19] Willis WD, Coggeshall RE. Sensory mechanisms of the spinal cord. 3rd Edition, New York, Plenum, 2004; pp. 19-90.

[20] Chaplan SR, Bach FW, Pogrel JW, Chung JM, Yaksh TL. Quantitative assessment of tactile allodynia in the rat paw. J Neurosci Methods 1994; 53(1): 55-63.

[http://dx.doi.org/10.1016/0165-0270(94)90144-9] [PMID: 7990513]

[21] Ren Y, Zou X, Fang L, Lin Q. Involvement of peripheral purinoceptors in sympathetic modulation of capsaicin-induced sensitization of primary afferent fibers. J Neurophysiol 2006; 96(5): 2207-16.

[http://dx.doi.org/10.1152/jn.00502.2006] [PMID: 16885522]

[22] Hathway GJ, Vega-Avelaira D, Moss A, et al. Brief, low frequency stimulation of rat peripheral C-fibres evokes prolonged microglialinduced central sensitization in adults but not in neonates. Pain 2009; 144: 110-8.

[23] Chahl LA, Ladd RJ. Local oedema and general excitation of cutaneous sensory receptors produced by electrical stimulation of the saphenous nerve in the rat. Pain 1976; 2: 25-34.

[24] Fitzgerald M. The spread of sensitization of polymodal nociceptors in the rabbit from nearby injury and by antidromic nerve stimulation. J Physiol 1979; 297(0): 207-16.

[http://dx.doi.org/10.1113/jphysiol.1979.sp013035] [PMID: 536911]

[25] Panopoulos P, Gazelius B, Olgart L. Alterations in intradental nerve responsiveness induced by electrical stimulation of the inferior alveolar nerve in the cat. Acta Physiol Scand Suppl 1983; 515: 37-43. [PMID: 6194654]

[26] Reeh PW, Kocher L, Jung S. Does neurogenic inflammation alter the sensitivity of unmyelinated nociceptors in the rat? Brain Res 1986; 384(1): 42-50.

[http://dx.doi.org/10.1016/0006-8993(86)91217-5] [PMID: 3790998]

[27] Meyer RA, Campbell JN, Raja SN. Antidromic nerve stimulation in monkey does not sensitize unmyelinated nociceptors to heat. Brain Res 1988; 441(1-2): 168-72.

[http://dx.doi.org/10.1016/0006-8993(88)91395-9] [PMID: 3359229]

[28] Dai Y, Iwata K, Fukuoka T, et al. Phosphorylation of extracellular signal-regulated kinase in primary afferent neurons by noxious stimuli and its involvement in peripheral sensitization. J Neurosci 2002; 22(17): 7737-45.

[http://dx.doi.org/10.1523/JNEUROSCI.22-17-07737.2002] [PMID: 12196597]

[29] Ferrari LF, Bogen O, Reichling DB, Levine JD. Accounting for the delay in the transition from acute to chronic pain: axonal and nuclear mechanisms. J Neurosci 2015; 35(2): 495-507.

[http://dx.doi.org/10.1523/JNEUROSCI.5147-13.2015] [PMID: 2558 9745]

[30] Zanjani TM, Sabetkasaei M, Mosaffa N, Manaheji H, Labibi F, Farokhi B. Suppression of interleukin- 6 by minocycline in a rat model of neuropathic pain. Eur J Pharmacol 2006; 538(1-3): 66-72. [http://dx.doi.org/10.1016/j.ejphar.2006.03.063] [PMID: 16687137]

[31] Hanani M. Satellite glial cells in sensory ganglia: From form to function. Brain Res Rev 2005; 48: 457-76.

[32] Zhang X, Chen Y, Wang C, Huang LY. Neuronal somatic ATP release triggers neuron-satellite glial cell communication in dorsal root ganglia. Proc Natl Acad Sci USA 2007; 104(23): 9864-9. [http://dx.doi.org/10.1073/pnas.0611048104] [PMID: 17525149]

[33] Hanani M. Intercellular communication in sensory ganglia by purinergic receptors and gap junctions: implications for chronic pain. Brain Res 2012; 1487: 183-91.

[http://dx.doi.org/10.1016/j.brainres.2012.03.070] [PMID: 22771859]

[34] Wagner L, Warwick RA, Pannicke T, Reichenbach A, Grosche A, Hanani M. Glutamate release from satellite glial cells of the murine trigeminal ganglion. Neurosci Lett 2014; 578: 143-7. [http://dx.doi.org/10.1016/j.neulet.2014.06.047] [PMID: 24993296]

[35] Ferrari LF, Lotufo CM, Araldi D, et al. Inflammatory sensitization of nociceptors depends on activation of NMDA receptors in DRG satellite cells. Proc Natl Acad Sci USA 2014; 111(51): 18363-8. [http://dx.doi.org/10.1073/pnas.1420601111] [PMID: 25489099]

\section{(C) 2019 Gong and Lin.}

This is an open access article distributed under the terms of the Creative Commons Attribution 4.0 International Public License (CC-BY 4.0), a copy of which is available at: (https://creativecommons.org/licenses/by/4.0/legalcode). This license permits unrestricted use, distribution, and reproduction in any medium, provided the original author and source are credited. 